\title{
ON SOLUTIONS OF RIEMANN'S FUNCTIONAL EQUATION
}

\author{
BY K. CHANDRASEKHARAN AND S. MANDELBROJT
}

Communicated by S. Bochner, August 17, 1959

1. Let $\left\{\lambda_{n}\right\},\left\{\mu_{n}\right\}(n \geqq 1)$ be two given sequences of positive numbers increasing to infinity, and let $\delta>0$. We call the triplet $\left\{\delta, \lambda_{n}, \mu_{n}\right\}$ a label. If $s$ is a complex variable, $s=\sigma+i \tau$, we speak of a solution of Riemann's functional equation

$$
\pi^{-s / 2} \Gamma\left(\frac{1}{2} s\right) \phi(s)=\pi^{-(\delta-s) / 2} \Gamma\left\{\frac{1}{2}(\delta-s)\right\} \psi(\delta-s),
$$

pertaining to the label $\left\{\delta, \lambda_{n}, \mu_{n}\right\}$, if there exist two Dirichlet series $\phi(s)=\sum a_{n} \lambda_{n}^{-s}, \psi(s)=\sum b_{n} \mu_{n}^{-s}\left(a_{n}\right.$ and $b_{n}$ complex) which do not vanish identically, and which admit finite abscissae of absolute convergence, and a function $\chi(s)$ which is holomorphic and uniform in a domain $|s|>R$, such that $\lim _{|\tau| \rightarrow \infty} \chi(\sigma+i \tau)=0$ uniformly in every segment $\sigma_{1} \leqq \sigma \leqq \sigma_{2}$, and such that, for some pair of real numbers $\alpha, \beta$, we have

$$
\chi(s)= \begin{cases}\pi^{-8 / 2} \Gamma\left(\frac{1}{2} s\right) \phi(s), & \text { for } \sigma>\alpha, \\ \pi^{-(\delta-s) / 2} \Gamma\left\{\frac{1}{2}(\delta-s)\right\} \psi(\delta-s), & \text { for } \sigma<\beta .\end{cases}
$$

In three papers published recently, Bochner and Chandrasekharan [2], Chandrasekharan and Mandelbrojt [3], and Kahane and Mandelbrojt [4], have studied the problem of finding an upper bound for the number of linearly independent solutions of equation (1.1). Their results enable one to establish in certain cases a unique solution, and in certain others to deduce that the sequences $\left\{\lambda_{n}\right\},\left\{\mu_{n}\right\}$ are periodic. In this note, which is a sequel to [3], we shall consider certain simple conditions which would ensure that $\delta=1$. Let

$$
D^{\mu}=\lim \sup \left(n / \mu_{n}\right), \quad h_{\mu}=\lim \inf \left(\mu_{n+1}-\mu_{n}\right) .
$$

We prove the following results.

THEOREM 1. If $h_{\lambda} \cdot h_{\mu}=1, \delta$ is an odd integer, and equation (1.1) has $a$ solution, then $\lambda_{n+1}-\lambda_{n}=h_{\lambda}$, and $\mu_{n+1}-\mu_{n}=h_{\mu}$, for every $n \geqq 1$. In particular, if $h_{\lambda}=h_{\mu}=1, \delta$ is an odd integer, and equation (1.1) has a solution, then $\lambda_{n+1}-\lambda_{n}=1$, and $\mu_{n+1}-\mu_{n}=1$ for every $n \geqq 1$. 
THEOREM 2. If $h_{\mu}>0, \delta$ is an odd integer, $b_{n}=O(1)$, and equation (1.1) has a solution, then $\delta=1$.

Theorem 3. Let $h_{\mu}>0$, and let $\delta$ be an odd integer. If simultaneously, $\left(\sum a_{n} \lambda_{n}^{-s}, \sum b_{n} \mu_{n}^{-s}\right)$ is a solution of equation (1.1) with the label $\left(\delta, \lambda_{n}, \mu_{n}\right)$, and $\left(\sum c_{n} \lambda_{n}^{\prime-s}, \sum d_{n} \mu_{n}^{-s}\right)$ is a solution with the label $\left(\delta, \lambda_{n}^{\prime}, \mu_{n}\right)$ for some $\left(\lambda_{n}^{\prime}\right)$, and $\left(\sum e_{n} \lambda_{n}^{\prime \prime-s}, \sum b_{n} d_{n} \mu_{n}^{-s}\right)$ is also a solution with the label $\left(\delta, \lambda_{n}^{\prime \prime}, \mu_{n}\right)$ for some $\left(\lambda_{n}^{\prime \prime}\right)$, where $\left(b_{n} / d_{n}\right)=o\left(\mu_{n}\right)$; then $\delta=1$.

2. For the proof of these theorems we require a number of lemmas.

LEMma 1. Equation (1.1) implies, for a sufficiently large integer $r$, the following relation:

$$
\begin{aligned}
& \Gamma\left\{\frac{1}{2}(\delta+1)\right\} \pi^{-(\delta+1) / 2} \sum_{n=1}^{\infty} a_{n} {\left[\frac{d^{2 r}}{d s^{2 r}} \frac{s}{\left(s^{2}+\lambda_{n}^{2}\right)^{(\delta+1) / 2}}\right]-K_{r}(s) } \\
&=(2 \pi)^{2 r} \sum_{n=1}^{\infty} b_{n} \mu_{n}^{2 r} \exp \left(-2 \pi \mu_{n} s\right),
\end{aligned}
$$

for $\operatorname{Re} s>0$, where $K_{r}(s)$ is holomorphic on the surface on which $\log s$ is defined, and $K_{r}(s)=O\left(|s|^{-\epsilon}\right), \epsilon>0$, as $s \rightarrow \infty$ in any angle $|\arg s|$ $\leqq \theta_{0}$.

This has been pruved by Bochner and Chandrasekharan [Theorem 2.1, p. 344]. By the definition of functional equation (1.1) it follows that the Dirichlet series on the right of (2.1) converges absolutely for $\sigma>0$, and from (2.1) it follows that the singularities of its sumfunction are situated symmetrically on the imaginary axis $\sigma=0$, at the points $\left( \pm i \lambda_{n}\right)$, and also possibly at the origin, which we may, for convenience, designate as $\lambda_{0}$.

Lemma 2. If $D^{\mu}<\infty$, and equation (1.1) has a solution, then $D^{\lambda} \cdot D^{\mu}$ $\geqq 1$, and $h_{\lambda} \cdot h_{\mu} \leqq 1$. (With the understanding that if $D^{\mu}=0$, then $D^{\lambda}$ $=+\infty$.)

This is an immediate consequence of a theorem of Chandrasekharan and Mandelbrojt [3, Theorem 1, p. 289] which implies [loc. cit., p. 290, 11. 6-9] that if $D^{\mu}<\infty$, and equation (1.1) is satisfied, then $\lambda_{n+1}-\lambda_{n} \leqq D^{\mu}$ for every $n \geqq 1$, that is, $\lambda_{n} \leqq n \cdot D^{\mu}$, or $n / \lambda_{n} \geqq 1 / D^{\mu}$, or $D^{\lambda} \cdot D^{\mu} \geqq 1$. Since we have $D^{\mu} \cdot h_{\mu} \leqq 1$, it follows that $h_{\lambda} \cdot h_{\mu} \leqq 1$.

LEMMA 3. If $h_{\mu}>0, \delta$ is an odd integer, and equation (1.1) has a solution, then $\delta=1$ or 3 .

This is a result of Kahane and Mandelbrojt [4, Theorem 3, pp. 71-72]. 
LEMMA 4. If $h_{\mu}>0$, and $\delta=1$ or 3 , and equation (1.1) has a solution, then $\mu_{n+1}-\mu_{n} \geqq h_{\mu}$. And for $\sigma<0$, the analytic continuation of the series

$$
\Psi(s)= \begin{cases}\sum b_{n} \exp \left(-2 \pi \mu_{n} s\right) & \text { if } \delta=1, \\ \sum b_{n} \mu_{n}^{-1} \exp \left(-2 \pi \mu_{n} s\right), & \text { if } \delta=3,\end{cases}
$$

which is a uniform function, is given by the series $-\sum_{1}^{\infty} b_{n} \exp \left(+2 \pi \mu_{n} s\right)$, and the only singularities of $\Psi(s)$ are simple poles at the points $\pm i \lambda_{n}$, $n=0,1,2, \cdots$.

A result proved earlier by Chandrasekharan and Mandelbrojt [3, Theorem 3, p. 292] gives the Dirichlet series representation of $\Psi(s)$ in the negative half-plane as $\sum c_{n} \exp \left(2 \pi \mu_{n}^{\prime} s\right)$ but it is easy to see that $c_{n}=-b_{n}$, and $\mu_{n}=\mu_{n}^{\prime}$, if one observes that by Agmon's theorem, used in that proof, the origin is a simple pole for the residual function $K_{r}(s)$ in (2.1). This fact is also obvious from the paper by Kahane and Mandelbrojt [4].

Lemma 5. If $h_{\mu}>0$, and $f(s)=\sum_{0}^{\infty} B_{n} \exp \left(-2 \pi \mu_{n} s\right)$ has $\sigma=0$ as its abscissa of absolute convergence, and the only singularities of $f(s)$ on a segment of the imaginary axis of length greater than $h_{\mu}^{-1}$ are poles of greatest order $q$, then $B_{n}=O\left(\mu_{n}^{g-1}\right)$.

This is a tauberian theorem of S. Agmon [1, Theorem 4.3(C)].

LemмA 6. If $D^{\mu}<\infty$, and $b_{n}=O\left(\mu_{n}^{q-1}\right)$, then for $\sigma>0$, we have

$$
f(s) \equiv \sum_{1}^{\infty} b_{n} \exp \left(-2 \pi \mu_{n} s\right)=O\left(\sigma^{-q}\right)
$$

If in the hypothesis we have $b_{n}=o\left(\mu_{n}^{q-1}\right)$, then the conclusion is $f(s)$ $=o\left(\sigma^{-q}\right)$.

(i) Since $D^{\mu}<\infty$, we have $\mu_{n}>L n$ for every $n$, where $L$ is some constant. Now, for $\sigma>0$, we have

$$
\begin{aligned}
|f(s)| & \leqq C \cdot \sum_{1}^{\infty} \mu_{n}^{q-1} \exp \left(-2 \pi \mu_{n} \sigma\right) \\
& \leqq C \cdot(2 \pi \sigma)^{1-q} \sum_{1}^{\infty}\left(2 \pi \mu_{n} \sigma\right)^{q-1} \exp \left(-2 \pi \mu_{n} \sigma\right) .
\end{aligned}
$$

The term $\left(2 \pi \mu_{n} \sigma\right)^{q-1} \exp \left(-2 \pi \mu_{n} \sigma\right)$ decreases (as $\mu_{n}$ increases), when $2 \pi \mu_{n} \sigma>q-1$. Let $n_{\sigma}$ be the smallest $n$ for which we have $2 \pi L n \sigma$ $>q-1$; in other words, for $n=1, \cdots, n_{\sigma}-1$, we have $2 \pi \operatorname{Ln} \sigma \leqq q-1$. Then 


$$
\begin{aligned}
\sum_{n_{\sigma}}^{\infty}\left(2 \pi \mu_{n} \sigma\right)^{q-1} \exp \left(-2 \pi \mu_{n} \sigma\right) & \leqq \sum_{n_{\sigma}}^{\infty}(L \cdot 2 \pi n \sigma)^{q-1} \exp (-2 \pi L n \sigma) \\
& =O\left(\sigma^{q-1} \sum_{n_{\sigma}}^{\infty} n^{q-1} \exp (-2 \pi L n \sigma)\right) \\
& =O\left(\sigma^{-1}\right)
\end{aligned}
$$

while

$$
\begin{aligned}
\sum_{1}^{n_{\sigma}-1}\left(2 \pi \mu_{n} \sigma\right)^{q-1} \exp \left(-2 \pi \mu_{n} \sigma\right) & \leqq \max _{x \geqq 0}\left[x^{q-1} e^{-x}\right] \cdot\left(n_{\sigma}-1\right) \\
& \leqq K \cdot\left(n_{\sigma}-1\right)=O\left(\sigma^{-1}\right)
\end{aligned}
$$

Hence $f(s)=O\left(\sigma^{-q}\right)$.

(ii) In case $b_{n}=o\left(\mu_{n}^{q-1}\right)$, let $n_{\sigma}$ be the smallest $n$ such that $(2 \pi n \sigma)$ $>(q-1) \sigma^{1 / 2}$. Then, as before,

$$
\left|\sum_{1}^{n_{\sigma}-1} b_{n} \exp \left(-2 \pi \mu_{n} s\right)\right|=O\left(\sigma^{1-q}\left(n_{\sigma}-1\right)\right)=O\left(\sigma^{1 / 2-q}\right),
$$

and, since $n_{\sigma} \rightarrow \infty$, as $\sigma \rightarrow 0$, we have

$$
\begin{aligned}
\left|\sum_{n_{\sigma}}^{\infty} b_{n} \exp \left(-2 \pi \mu_{n} s\right)\right| & =o\left(\sigma^{1-q}\right) \cdot\left|\sum_{n_{\sigma}}^{\infty}\left(2 \pi \mu_{n} \sigma\right)^{q-1} \exp \left(-2 \pi \mu_{n} \sigma\right)\right| \\
& =o\left(\sigma^{-q}\right) .
\end{aligned}
$$

Hence $f(s)=o\left(\sigma^{-q}\right)$.

3. We shall now indicate the proofs of Theorem 1 to 3 .

Proof of Theorem 1. We remark that by Lemma 2 , we have $h_{\lambda} \cdot h_{\mu} \leqq 1$. If $h_{\lambda} \cdot h_{\mu}=1$, then we have $h_{\lambda}>0$, and $h_{\mu}>0$, so that $D^{\lambda}<\infty$, and $D^{\mu}<\infty$. Hence, as in the proof of Lemma 2, we have $\mu_{n+1}-\mu_{n}$ $\leqq D^{\lambda} \leqq h_{\lambda}^{-1}=h_{\mu}$, and $\lambda_{n+1}-\lambda_{n} \leqq D^{\mu} \leqq h_{\mu}^{-1}=h_{\lambda}$. Since $\delta$ is odd, we have, by Lemma $3, \delta=1$ or 3 . Now, by the first part of Lemma 4 , we have $\mu_{n+1}-\mu_{n} \geqq h_{\mu}$, and $\lambda_{n+1}-\lambda_{n} \geqq h_{\lambda}$, which lead to the desired result.

Proof of Theorem 2. By Lemma 3 , we have $\delta=1$ or 3 . We shall show that the case $\delta=3$ is incompatible with the hypotheses. Consider the series $f(s)=\sum b_{n} \mu_{n}^{2 r} \exp \left(-2 \pi \mu_{n} s\right)$ in Lemma 1. Since $b_{n}=O(1)$, we have, by Lemma $6, f(s)=O\left(\sigma^{-2 r-1}\right)$, for $\sigma>0$. On the other hand, in a neighborhood of a pole, say $s=i \lambda_{n}, n \geqq 1$, we have $|f(s)|>c \cdot|\sigma|^{-p}$, where $p$ is the order of the pole, hence an integer, with $p=(1 / 2)(\delta+1)+2 r$. For these two estimates to be compatible, we should have $\delta=1$.

Proof of Theorem 3. It is sufficient to show that $\delta=3$ is impos- 
sible. If $\delta=3$, then by Lemmas 5 and 1 , we have $b_{n}=O\left(\mu_{n}\right), d_{n}=O\left(\mu_{n}\right)$ and $b_{n} d_{n}=O\left(\mu_{n}\right)$. But by hypothesis, $\left|b_{n}\right| \leqq \epsilon_{n} \cdot\left|d_{n}\right| \cdot \mu_{n}$, where $\epsilon_{n}>0$, and $\epsilon_{n} \rightarrow 0$ as $n \rightarrow \infty$. That is, $\left|b_{n} d_{n}\right| \geqq\left|b_{n}\right|^{2} \cdot\left(\mu_{n} \epsilon_{n}\right)^{-1}$. We now observe that $b_{n}=o\left(\mu_{n}\right)$ is impossible, because otherwise, by Lemma 6(ii), we should have $f(s)=o\left(\sigma^{-2}\right)$, which contradicts the fact that $\left|f\left(i \lambda_{n}+\sigma\right)\right|$ $>c \cdot \sigma^{-2}$ for $\sigma>0$. Hence there exists a sequence $\left(n_{j}\right)$ such that $\left|b_{n_{j}}\right|>\epsilon_{n_{j}}^{1 / 3} \cdot \mu_{n_{j}}$, which, together with the inequality for $b_{n} d_{n}$ obtained above, yields $\left|b_{n_{j}} \cdot d_{n_{j}}\right| \geqq \epsilon_{n_{j}}^{2 / 3} \cdot \mu_{n_{j}}^{2} \cdot\left(\mu_{n_{j}} \cdot \epsilon_{n_{j}}\right)^{-1} \geqq \mu_{n_{j}} \cdot \epsilon_{n_{j}}^{-1 / 3}$. But this contradicts the fact that $b_{n} d_{n}=O\left(\mu_{n}\right)$.

\section{REFERENCES}

1. S. Agmon, Complex variable Tauberians, Trans. Amer. Math. Soc. vol. 74 (1953) pp. 444-481.

2. S. Bochner and K. Chandrasekharan, On Riemann's functional equation, Ann. of Math. vol. 63 (1956) pp. 336-360.

3. K. Chandrasekharan and S. Mandelbrojt, On Riemann's functional equation, Ann. of Math. vol. 66 (1957) pp. 285-296.

4. J. P. Kahane and S. Mandelbrojt, Sur l'equation functionnelle de Riemann et la formule sommatoire de Poisson, Ann. Sci. Ecole Norm. Sup. vol. 65 (1958) pp. 57-80.

Tata Institute of Fundamental Research, Bombay and College de France, Paris 